\title{
Evidências da contribuição dos programas de assistência ao idoso na promoção do envelhecimento saudável no Brasil
}

\author{
Larissa Fortunato Araújo, ${ }^{1}$ Carolina Gomes Coelho, ${ }^{1}$ \\ Érica Toledo de Mendonça, ${ }^{2}$ Aline Vasconcellos Martins Vaz, ${ }^{3}$ \\ Rodrigo Siqueira-Batista ${ }^{2}$ e Rosângela Minardi Mitre Cotta ${ }^{4}$
}

Como citar Araújo LF, Coelho CG, de Mendonça ET, Vaz AVM, Siqueira-Batista R, Cotta RMM. Evidências da contribuição dos programas de assistência ao idoso na promoção do envelhecimento saudável no Brasil. Rev Panam Salud Publica. 2011:30(1):80-6.

RESUMO Objetivo. Buscar na literatura evidências relativas à contribuição dos programas de promoção da saúde no processo de envelhecimento saudável no Brasil.

Métodos. Pesquisaram-se as bases de dados Science Direct, SciELO, LILACS, IBECS, Biblioteca Cochrane e MEDLINE em busca de artigos que enfocassem iniciativas voltadas para a população idosa e o envelhecimento saudável, que avaliassem multidimensionalmente a qualidade de vida do idoso e que tratassem de aspectos relativos às práticas profissionais especificas em promoção da saúde. Para definir a eficácia das iniciativas, adotaram-se os seguintes critérios: ter metas que incluíssem a melhoria da saúde e do bem-estar; ter foco na saúde holística; usar o empoderamento como mecanismo central; atender os pré-requisitos da saúde e realizar ações nas áreas prioritárias da Carta de Ottawa.

Resultados. Foram identificados 10 artigos que se referiam a cinco programas de promoção da saúde do idoso no Brasil. Pode-se afirmar que todos os programas apresentados demonstraram elementos que vão ao encontro da promoção da saúde no envelhecimento por abranger características que permitem a inserção dos idosos na sociedade por meio da criação do ambiente de suporte para prevenção de agravos, aumento da capacidade funcional e melhoria da qualidade de vida, tendo sido atendidos os critérios de eficácia.

Conclusões. É preciso produzir evidências científicas acerca da efetividade, eficiência e eficácia dos programas para a terceira idade para que se possa avaliar o valor dessas ações na promoção da saúde no envelhecimento.

Palavras-chave Prática clínica baseada em evidências; promoção da saúde; envelhecimento; Brasil.

A população idosa vem crescendo em diferentes regiões do mundo, detectando-

\footnotetext{
Universidade Federal de Viçosa, Departamento de Nutrição e Saúde, Viçosa (MG), Brasil.

2 Universidade Federal de Viçosa, Departamento de Medicina e Enfermagem (DEM), Viçosa (MG), Brasil.

3 Prefeitura Municipal de Viçosa (MG), Brasil.

4 Universidade Federal de Viçosa, Departamento de Nutrição e Saúde, Programa de Inovação em Docência Universitária (PRODUS), e Laboratório de Estudos em Planejamento e Gestão em Saúde, Viçosa (MG), Brasil. Correspondência: rmmitre@ufv.br
}

se, no entanto, significativas variações na velocidade desse crescimento. No Brasil, as modificações têm transcorrido de forma veloz em uma sociedade pouco preparada para tal transição (1): o número de idosos passou dos 2 milhões em 1950 para 15,4 milhões em 2002, um aumento de $700 \%$ (2). As projeções indicam que, em 2025, o Brasil terá a sexta maior população mundial de idosos, correspondendo a aproximadamente $15 \%$ do povo brasileiro, ou seja, aproximadamente 30 milhões de pessoas (3).

Com efeito, o envelhecimento populacional, fruto de conquistas nos âmbitos científico, tecnológico e social, tornou-se um grande desafio para as políticas públicas e os setores sociais, gerando um grande impacto nos custos da saúde (4, 5). A Política Nacional de Promoção da Saúde, inscrita no Pacto pela Saúde, ratifica o compromisso do Ministério da 
TABELA 1. Descritores utilizados na busca de referências bibliográficas sobre programas de promoção da saúde do idoso no Brasil, $2010^{a}$

\begin{tabular}{|c|c|c|}
\hline Português & Inglês & Espanhol \\
\hline Idosos + programas & Elderly + program & Personas mayores + programa \\
\hline Idosos + promoção da saúde & Elderly + health promotion & Personas mayores + promoción de la salud \\
\hline Idosos + programas + promoção da saúde & Elderly + program + health promotion & $\begin{array}{l}\text { Personas mayores + programas + promoción de la } \\
\quad \text { salud }\end{array}$ \\
\hline Idosos + programas + promoção da saúde + eficácia & Elderly + program + health promotion + effectiveness & $\begin{array}{l}\text { Personas mayores + programa + promoción de la } \\
\text { salud + eficacia }\end{array}$ \\
\hline $\begin{array}{l}\text { Idosos + programas + promoção da saúde + } \\
\text { avaliação }\end{array}$ & Elderly + program + health promotion + evaluation & $\begin{array}{l}\text { Personas mayores + programa + promoción de la } \\
\text { salud + evaluación }\end{array}$ \\
\hline $\begin{array}{l}\text { Idosos + programas + promoção da saúde + eficácia } \\
\quad+\text { avaliação }\end{array}$ & $\begin{array}{l}\text { Elderly + program + health promotion + effectiveness } \\
+ \text { evaluation }\end{array}$ & $\begin{array}{l}\text { Personas mayores + programa + promoción de la } \\
\text { salud + eficacia + evaluación }\end{array}$ \\
\hline
\end{tabular}

a Foram pesquisadas as bases Science Direct, SciELO, LILACS, IBECS, Biblioteca Cochrane e MEDLINE.

Saúde do Brasil com a ampliação e a qualificação das ações de promoção da saúde nos serviços e na gestão do Sistema Único de Saúde (SUS). O Pacto pela Vida, que está contido no Pacto pela Saúde, contém, entre os objetivos e metas prioritárias, a atenção ao idoso (6).

Assim, a portaria 2 528, de 19 de outubro de 2006, que regulamenta a Política Nacional de Saúde da Pessoa Idosa, expõe que "quando o processo de envelhecer é aceito como um êxito, o aproveitamento da competência, da experiência e dos recursos humanos dos grupos mais velhos é assumido com naturalidade, como uma vantagem para o crescimento de sociedades humanas maduras e plenamente integradas" (6). Nessa perspectiva, o envelhecimento bem-sucedido pode ser entendido a partir de três componentes: a) menor probabilidade de doença, b) alta capacidade funcional física e mental e c) engajamento social ativo junto à teia social. O alcance desses fatores requer a promoção do envelhecimento com qualidade de vida, enfatizando-se os aspectos preventivos e assistenciais de maior relevância entre a população idosa (5).

Em um contexto de envelhecimento populacional, os programas de promoção da saúde do idoso são cada vez mais necessários. Atualmente, a maioria dos programas está no âmbito público ou da extensão universitária. Em geral, os programas convergem em ações que privilegiam o envelhecimento ativo, com foco em uma longevidade que preserva a capacidade funcional e o estímulo à autonomia (7). Entretanto, embora haja relatos dessa experiência na literatura, não há estudos no Brasil que mostrem evidências consistentes de seus benefícios por meio de avaliações. O objetivo do presente estudo foi buscar na literatura evidências relativas à contribuição dos programas de promoção da saúde para o processo de envelhecimento saudável no Brasil, através da análise e avaliação das ações desenvolvidas pelos mesmos.

\section{MÉTODOS}

Realizou-se em 2010 uma revisão de literatura nas bases de dados Science Direct (http:/ /www.sciencedirect.com/), SciELO (http://www.scielo.org/php/ index.php), Literatura Latino-Americana e do Caribe em Ciências da Saúde (LILACS) (http:/ / lilacs.bvsalud.org), Índice Bibliográfico Espanhol de Ciências de Saúde (IBECS) (http:/ /ibecs.isciii.es/ cgi-bin/wxislind.exe/iah/online/?Isis Script $=\mathrm{iah} / \mathrm{iah}$.xis\&base $=$ IBECS\&lang=e), Biblioteca Cochrane (http://cochrane. bvsalud.org/portal/php/index.php) e MEDLINE (http://www.ncbi.nlm.nih. gov/pubmed/). Os descritores utilizados na busca de dados, nos idiomas português, inglês e espanhol estão apresentados na tabela 1.

A partir da leitura dos resumos identificados, foram selecionados os artigos que apresentaram iniciativas brasileiras com os seguintes elementos estabelecidos por Assis et al. (7): a) foco na população idosa; b) discussão conceitual ou institucional sobre promoção da saúde; c) foco no envelhecimento saudável, apoio social e saúde do idoso; d) avaliação multidimensional/estudos epidemiológicos sobre qualidade de vida do idoso; e) aspectos relativos às práticas profissionais específicas em promoção da saúde.

Para definir a eficácia dos programas de promoção da saúde para a terceira idade descritos na literatura, adotaram-se os critérios relacionadas por Goodstadt et al. (8): a) ter metas que vão além da prevenção da morbidade, incluindo a melho- ria da saúde e do bem-estar; b) ter como foco a saúde positiva, holística, a justiça social, a equidade e participação; c) usar o empoderamento como mecanismo central; d) atender os pré-requisitos da saúde (fatores de nível macrossocial); e) realizar ações nas áreas prioritárias da Carta de Ottawa (ação comunitária, construção de políticas públicas saudáveis, criação de ambientes de suporte, desenvolvimento de habilidades pessoais e reorientação dos serviços de saúde).

Dessa forma, considerou-se como eficaz o programa que demonstrou impacto no bem-estar e na saúde dos idosos além de oferecer um levantamento, realizado pelos próprios idealizadores dos programas ou por órgãos públicos capacitados, dos pontos positivos e negativos das ações de promoção de saúde.

\section{RESULTADOS}

Foram pesquisadas referências relativas ao período de 1994 a 2010, sendo identificados 11751 estudos. Após leitura dos títulos dos trabalhos encontrados, procedeu-se a uma nova seleção, na qual realizou-se uma leitura dos resumos que continham informações de interesse para o estudo. Desse total, foram selecionados 10 trabalhos, entre artigos, teses, dissertações e livros, que atendiam aos objetivos propostos e aos critérios adotados por Goodstadt et al. (8), e abordavam a promoção da saúde do idoso em cinco programas existentes no Brasil: Projeto de Valorização do Envelhecer (PROVE) (9), Programa Interdisciplinar de apoio à Terceira Idade (PIATI) (10, 11), Grupo de Atenção à Saúde do Idoso (GRASI) (12) e Universidade Aberta para a Terceira Idade (UnATI) do Rio de Janeiro $(7,8,13-15)$ e de Pernambuco (16). 
O PROVE é desenvolvido desde 1996 no Instituto de Neurologia Deolindo Couto, na Universidade Federal do Rio de Janeiro (UFRJ). O trabalho realizado é multidisciplinar e multidimensional e tem como base a pesquisa qualitativa. Sua atividade primordial são os grupos de encontro de idosos. Esses grupos trabalham com informação e reflexão sobre processos ligados ao envelhecimento, ou seja, têm como metodologia de trabalho as palestras de orientação para a saúde, baseadas no programa do Ministério da Saúde "Viva bem a idade que você tem" (9). O projeto oferece atividades como acolhimento de idosos recém-ingressos no projeto e oficinas de dança, poesia, pintura, alongamento, trabalho de movimentação e estimulação psicomotora. Dados da pesquisa qualitativa realizada por Teixeira (9) informam que os idosos se sentiam realizados com o fato de participar desses encontros, sendo que suas falas, registradas no artigo, mostram que o envelhecimento pode ser um momento de construção de conhecimentos e de aquisição de novas experiências de vida.

Outro programa identificado na literatura foi o PIATI, que surgiu em 1999, vinculado à Universidade Federal de Campina Grande (UFCG), no Estado da Paraíba. Entre seus objetivos está o de qualificar o corpo docente e o corpo discente envolvidos nos projetos, através de minicursos sobre geriatria e gerontologia, contribuindo para a reorientação dos serviços de saúde (10). As atividades e propósitos do PIATI, tidos como metas para o programa, são: a) promover ações pedagógicas que incluam a melhoria da qualidade de vida do idoso, com ênfase na proteção à saúde, na educação continuada (alfabetização e escolarização); b) melhorar as relações familiares e intergeracionais e as práticas de sociabilidade; c) ampliar a consciência do idoso acerca do processo de envelhecimento; d) atuar junto aos grupos de convivência de idosos do município, colaborando com seus programas educativos de saúde e lazer; e) influenciar a melhoria das relações familiares e intergeracionais dos idosos e das outras gerações com os idosos; f) apoiar ações pedagógicas de cuidados com saúde e lúdicas para idosos asilados; g) conhecer o impacto da aplicação das políticas públicas implementadas pelo governo federal, estadual e municipal, junto ao segmento idoso da população (10). Este último propósito é condizente com uma avaliação, pois, para conhecer o impacto de políticas públicas, é necessário avaliá-las; entretanto, as conclusões sobre tal avaliação em nenhum momento foram citadas.

Por outro lado, o PIATI foi avaliado pelo programa de apoio à extensão universitária da Secretaria de Educação Superior do Ministério de Educação e Cultura (PROEXT/SESu/MEC 2003) como um dos melhores programas do país. Além disso, o trabalho do PIATI é avaliado continuamente após a conclusão do cronograma em cada grupo de idosos, através dos seguintes itens: participação dos sujeitos (frequência, atenção e interesse), cooperação e interesse nos questionamentos e atividades ofertadas, apreensão das informações e orientações ofertadas, adequação dos recursos didáticos, cumprimento das atividades programadas e nível de satisfação do idoso, sendo que esses dados, quantitativos ou qualitativos (relatos dos idosos), não estão disponíveis (11).

Outro programa, o GRASI, foi fundado em 1997, associado ao ambulatório de Clínica Médica do Hospital das Clínicas da Universidade Estadual de Campinas, no Município de Campinas, em São Paulo. A dinâmica do trabalho consiste em uma triagem dos idosos atendidos no ambulatório de Clínica Médica. Essa triagem serve para identificar o interesse dos idosos em participar do programa. A partir dos interessados, são formados quatro grupos. Cada grupo é constituído por cinco idosos e seus familiares, que participam de um programa educativo que compreende quatro encontros ao longo de 4 meses (um encontro mensal para cada grupo). O programa educativo aborda temas de interesse dos idosos e outros que foram considerados relevantes para o alcance dos objetivos propostos, como por exemplo, o processo de senescência e senilidade e fatores de risco e prevenção de quedas e de outros acidentes, com o uso de metodologias participativas e técnicas lúdicas (12). Na última reunião de cada grupo, é realizada uma avaliação geral, englobando todos os quatro encontros. Os autores (12) relatam que a maioria dos idosos e de seus acompanhantes gostam da experiência e aprendem coisas novas, pois manifestam interesse em divulgar as informações para amigos e familiares.

Na avaliação sobre a eficácia da implantação, a falta de assiduidade foi o maior problema enfrentado pelo GRASI, sendo que houve idosos que não par- ticiparam de todos os encontros de seu grupo. A experiência levou à reformulação do GRASI, com aumento do número de vagas de cinco para sete idosos em cada grupo e a modificação do conteúdo e das estratégias de apresentação. Foram introduzidos, por exemplo, jogos relativos a "memória e envelhecimento", tornando a atividade mais dinâmica e exigindo maior participação dos membros dos grupos (12).

A UnATI na UERJ encontra-se em permanente construção desde sua criação, em 1993, quando surgiu como um programa vinculado ao Instituto de Medicina Social. A partir de 1996, passou a incluir também o ambulatório do Núcleo de Atenção ao Idoso (NAI), que, além do atendimento ambulatorial, desenvolve projetos especiais, como o Projeto de Assistência e Estudos Cognitivos, o Programa de Atenção Integral ao Idoso Internado, o Projeto Promoção de Saúde, o Projeto Idoso em Movimento Mantendo a Autonomia e o Projeto Atendimento Domiciliar (13). Suas atividades socioculturais e educativas para a terceira idade se desenvolvem ao longo do ano todo. Até o presente momento, realizou cerca de 240 cursos gratuitos, oficinas, seminários, palestras, festas, exposições, shows de dança e música e programa de voluntariado. Sua estrutura é, por definição, considerada aberta - no sentido de não concluída-, posto que pretende ser dinâmica (14).

O Projeto de Promoção da Saúde da UnATI possui duas linhas de ação integradas: o Grupo Encontros com a Saúde (GES) e a Avaliação Multidimensional de Saúde e Qualidade de Vida (AMSQV). A atividade educativa dos grupos baseia-se no debate sobre temas de saúde no envelhecimento, e a avaliação multidimensional conjuga a pesquisa sobre saúde do idoso com ações preventivas, educativas e orientações assistenciais, em nível individual (7).

Os GES são espaços de informação e reflexão sobre aspectos relativos à saúde no envelhecimento. Abordam temas como possibilidades e limites do envelhecimento, alimentação, atividade física, estresse, sexualidade, memória, saúde oral, direitos dos idosos e noções sobre doenças comuns (hipertensão arterial, diabetes, alterações osteoarticulares e depressão) (8). A AMSQV é utilizada para ações preventivas e educativas em nível individual, sendo aplicada simultaneamente à participação dos idosos nos gru- 
pos. Na dimensão da pesquisa, A AMSQV transcende o caráter de atividade-meio, ultrapassando o seu objetivo interventivo imediato e viabilizando a produção de conhecimento com alcance além da aplicabilidade local (7). O instrumento foi construído pela equipe interdisciplinar, tomando por base o questionário Brazilian Old Age Schedule (BOAS), desenvolvido a partir de outros instrumentos e adaptado para uso em pesquisas epidemiológicas com idosos no Brasil (7).

Segundo Fletcher et al. (14), uma avaliação multidimensional significa a realização de um inquérito sistemático, tanto quanto possível, de condições e problemas não espontaneamente trazidos à consulta. Trata-se de um método padrão para detectar problemas e, entre seus benefícios possíveis, está a identificação de questões desconhecidas cuja abordagem possibilite uma melhor saúde.

De acordo com Assis et al. (15), que avaliaram a eficácia da implantação da UnATI na UERJ, a influência do projeto sugere o alcance de objetivos intermediários, como a socialização e o debate de informações, reforço da autoestima e ampliação dos contatos sociais. Isso ocorre pela proposta participativa e pela interação nos grupos, que gera um sentimento de pertencimento, de "ter uma turma", algo valioso entre idosos, dado o ocasional afrouxamento dos vínculos sociais. Um limite a ponderar da avaliação realizada por Assis et al. (15) é a possível superestimação de certos dados na avaliação inicial (como, por exemplo, a ampliação de conhecimentos e a reflexão sobre saúde e envelhecimento, reforço do autoconhecimento e da autoestima, e a ampliação dos contatos e da rede social dos idosos), uma vez que a AMSQV foi aplicada após o início dos grupos, suscetível assim à sua influência. A avaliação desenvolvida segue um modelo aberto, desenhado como exercício de avaliar a própria dinâmica do trabalho educativo e assistencial em seu potencial de produção de conhecimento, tendo em vista as dificuldades para sistematização e avaliação nos serviços de saúde. A utilização de métodos quantitativos e qualitativos coerentes com os pressupostos da promoção da saúde é uma empreitada complexa, que, contudo, deve ser trilhada para permitir o avanço da promoção da saúde na dinâmica assistencial e o aprofundamento do debate sobre avaliação de ações de promoção da saúde no Brasil (15).
A UnATI da Universidade Federal do Pernambuco é outro exemplo. Em consonância com as recomendações da Política Nacional do Idoso, foi criado em setembro de 1996. Apresenta características e propostas de atuação na promoção da saúde dos idosos semelhantes às da UnATI/UERJ. Tendo como objetivo principal promover a integração entre universidade, idoso e comunidade, envolve vários segmentos da comunidade universitária e da comunidade em geral (16). As atividades de formação profissional abrangem o ensino sobre envelhecimento através da disciplina de gerontologia, ministrada pelo Departamento de Terapia Ocupacional, os grupos de estudo sobre envelhecimento e estágios curriculares. Os cursos são públicos e gratuitos e não há exigência de nível de escolaridade. Cada idoso só pode se inscrever em um curso, uma vez que a demanda tem sido maior que a oferta; porém, uma vez iniciadas as aulas, caso haja alguma vaga disponível, essa é novamente oferecida, de modo que aqueles que desejarem possam frequentar mais de um curso (16).

Em 1999, a UnATI em Pernambuco contava com cerca de 530 idosos cadastrados e 32 turmas, distribuídas como segue: relaxamento neuromuscular; natação; dinâmica dos corpos; hataioga; ioga para terceira idade; iniciação ao sistema de biodança; educação em saúde; bioenergética; primeiros socorros no local do acidente; nutrição e envelhecimento; plantas medicinais: conhecer para melhor utilizar; segurança no lar; reciclagem de papel e encadernação artesanal; oficina de artes; embalagem artesanal; papel vegetal; oficina de bijuteria; arte e criatividade; para gostar de ler; redação: aspectos diversos; oficina literária; introdução ao estudo da língua italiana; inglês; grupos interativos; técnicas de leitura; educação participativa; e informática básica (16). O fato de o programa permitir que os idosos frequentem o campus universitário é uma iniciativa viabilizadora da convivência entre pares e com outras gerações.

\section{DISCUSSÃO}

De acordo com a Primeira Conferência Internacional de Promoção da Saúde, realizada em Ottawa, no Canadá, em 1986, "Promoção da saúde é o nome dado ao processo de capacitação da comunidade para atuar na melhoria de sua qualidade de vida e saúde, incluindo uma maior participação no controle deste processo" (17). Corroborando esse conceito, indivíduos capacitados têm maior poder de interferir no seu próprio processo de saúde, adoecimento e doença, ampliando a sua visão acerca dos fatores relacionados à produção social da saúde.

Segundo Martins et al. (18), o compromisso da promoção da saúde com a produção de sujeitos reflexivos autônomos e socialmente solidários só é eficaz através do empoderamento, que consiste na ampliação das possibilidades de controle, por um sujeito ou uma população, dos aspectos significativos relacionados à sua própria existência $(19,20)$, sendo necessária também a viabilização desse compromisso por meio da criação de espaços e práticas democráticas no campo da saúde (18). Contudo, a promoção da saúde, nessa perspectiva, não gera efeitos imediatos. É necessário um determinado período de tempo antes que os efeitos de estratégias e processos de promoção da saúde comecem a mostrar resultados que possam ser mensurados, o que torna sua visibilidade mais difícil.

De acordo com a literatura encontrada, datam da década de 1970 os primeiros avanços na criação de uma base sólida de evidências da eficácia da promoção da saúde. Apesar da existência de lacunas importantes nesse campo do conhecimento, uma tarefa prioritária consiste em difundir melhor as evidências disponíveis sobre a eficácia para os gestores em saúde e para os profissionais da área da saúde, uma vez que esses são os responsáveis por sua aplicação (21). Com efeito, uma promoção da saúde eficaz conduz a mudanças nos determinantes da saúde e deve envolver a população no percurso do diagnóstico da situação e na avaliação das ações implantadas (22).

Constatamos, na literatura analisada, um número reduzido de produções científicas mostrando a avaliação e os resultados de programas de promoção da saúde no envelhecimento, tanto em nível nacional como internacional. Em geral, os artigos descreviam as atividades desenvolvidas em alguns programas, mas não avaliavam suas ações. Somente cinco programas puderam ser analisados e considerados como evidências de promoção da saúde no envelhecimento a partir do estabelecimento de critérios, como os descritos por Goodstadt et al. (8).

Apesar da escassez, na literatura, de evidências que comprovem a eficácia das ações de promoção da saúde no envelhe- 
cimento, os estudos abordados neste artigo demonstraram a importância dessas ações na melhoria da qualidade de vida dos idosos, uma vez que estimulam a participação, a interação, a integração e o empoderamento e libertação desses indivíduos. Ao se partir do pressuposto de que inúmeros programas de promoção da saúde destinados à terceira idade mostram claramente o benefício positivo para a saúde dessas pessoas, bem como para a sociedade, nota-se que o envelhecimento saudável, derivado do fato de os idosos manterem um estilo de vida saudável durante mais tempo, é revertido positivamente para a sociedade como um todo.

Os estudos de avaliação, com enfoque na análise da eficácia e eficiência de determinado programa, seriam apropriados para a análise ou mensuração do êxito técnico do programa, ou seja, de sua qualidade, através de métodos que empregam o entendimento da realidade a partir de uma ótica objetiva e do sucesso prático das ações de promoção da saúde propostas (23). Considerando tal fato, apesar de o conceito de eficiência se relacionar aos custos envolvidos nos programas sociais, tal aspecto não foi objeto de análise do presente estudo. Dessa forma, a análise da efetividade de um programa de saúde se propõe a desvendar os sentidos dos fenômenos, respeitando sua complexidade, riqueza e profundidade, além de considerar o universo que circunda os atores envolvidos, em especial os usuários a que se destinam as ações $(23,24)$.

No entanto, existe confusão acerca dos conceitos de eficácia, eficiência e efetividade. Assim, a eficácia confunde-se com a efetividade, porém não possui o "efeito" continuado das ações e práticas de saúde implementadas. Conforme Marinho e Façanha (25), a eficácia é a capacidade de obter o efeito que se deseja (os meios não são considerados); a eficiência é a capacidade de empregar da melhor maneira a relação entre os meios disponíveis para obtenção do efeito que se deseja, com dispêndio mínimo de recursos e esforços; e a efetividade é a capacidade que uma ação tem de ser continuada, de forma a manter ou melhorar seus resultados através do aprimoramento dos seus objetivos. O ideal seria que todos os programas de promoção da saúde fossem avaliados pela sua eficácia, eficiência e efetividade. Só assim seria possível aos responsáveis pelas políticas públicas atuarem dentro de estratégias mais concretas, pelo conheci- mento dos meios de promover a saúde com equidade para toda a população, viabilizando um direito, e não somente algo a ser adquirido.

Dessa forma, salienta-se a importância do controle dos resultados da aplicação de políticas públicas. Nesse controle, o ideal é partir da premissa de que uma gestão orientada por resultados tem mecanismos de aferição do desempenho, da satisfação do usuário e de controle social. Os programas com incentivos vinculados à produtividade e eficiência configuram as condições necessárias para a responsabilização dos gestores públicos. Não se trata de uma prestação de contas vinculada apenas a aspectos de natureza processual e legal, mas de uma prestação de contas que contemple, também, aspectos de eficiência, eficácia e efetividade da ação pública (26).

Os cinco programas apresentados neste artigo podem ser considerados como estratégias de promoção da saúde e ainda como propostas eficazes de intervenção. O PROVE proporcionou aos idosos meios para que se tornassem empoderados, e o entusiasmo e engajamento dos mesmos nos grupos que promovem a saúde fez com que houvesse uma ampliação de sua rede social. Portanto, esse projeto é uma estratégia de promoção da saúde, dado então como proposta eficiente de intervenção (9). Porém, o PROVE não foi avaliado na literatura. Portanto, apesar de se mostrar eficaz, não houve indícios de julgamento quanto ao sucesso prático dessa intervenção ao longo do tempo (9).

Em relação ao PIATI, no momento em que o projeto se propôs a criar oportunidades de ampliar os conhecimentos dos idosos, ofereceu uma valiosa contribuição para o empoderamento, melhorando a inserção social dos idosos e indo contra o processo de marginalização dos indivíduos de idade avançada na sociedade contemporânea. A estrutura descrita para o GRASI na literatura era eficaz para a promoção da saúde; porém, não foi encontrada nenhuma publicação posterior à sua implantação, e, portanto, não se sabe se o programa ainda está vigente ou se houve alterações nas estratégias de suas ações. Como não havia evidência científica de indicadores estatísticos, a eficácia do GRASI precisa ser medida por meio da qualidade. A evidência da eficácia do GRASI está nos relatos de casos dos idosos e de seus familiares sobre as melhoras alcançadas na saúde pela participação no programa. Eles relataram que gostaram e aprenderam coisas novas e importantes para o enfrentamento do dia a dia.

Sobre a UnATI do Rio de Janeiro, foram encontradas evidências científicas sobre a eficácia de suas ações de promoção de saúde vinculadas à UERJ. A eficácia do projeto da UERJ consiste na reorientação das práticas de saúde em direção a uma atenção integral, pautada pela humanização e pelo fortalecimento da participação dos sujeitos em questões que afetam a saúde e o bem-estar, no plano individual e coletivo.

A UnATI de Pernambuco possui como âncora a promoção da saúde nos mesmos moldes da UnATI na UERJ. Porém, a falta de evidência científica por meio de dados quantitativos e qualitativos resultantes de avaliação ou acompanhamento das estratégias de implementação à saúde dos idosos não consolida esse programa como eficiente, embora seja eficaz.

Ao mesmo tempo em que se percebe a necessidade de ampliação das políticas públicas para acolher um maior número de pessoas e, assim, viabilizar a continuidade das ações desenvolvidas (19), tornam-se necessários o estabelecimento e a consolidação de políticas públicas que contemplem, em seus desenhos metodológicos, a complexidade do envelhecimento saudável nas sociedades atuais. Sendo assim, é necessário avaliar a eficácia dessas ações a fim de verificar se os propósitos de tais políticas estão sendo cumpridos, promovendo efetivamente a saúde no envelhecimento. Para tal, as evidências científicas acerca dos programas para a terceira idade devem existir e ser identificadas, a fim de que também possam ser norteadoras de ações profissionais que vão atender, intervir e orientar o idoso sobre as maneiras pelas quais ele pode manter a saúde e a funcionalidade.

\section{CONSIDERAÇÕES FINAIS}

À luz da literatura consultada, nota-se que a adoção de programas de promoção da saúde voltados para o envelhecimento saudável representa uma estratégia eficaz para a melhoria da qualidade de vida e da saúde dos idosos.

Assim, a existência de serviços de atendimento aos idosos, vinculados, por exemplo, a instituições de ensino, como os serviços ambulatoriais, foram apresentados como alternativas para o atendimento integral à saúde do idoso, reali- 
zando intervenções e orientações, além de propiciar o desenvolvimento de práticas sociais de atenção primária em saúde, priorizando a educação em saúde baseada não só na perspectiva profissional, mas na perspectiva holística do sujeito.

Ao considerar os aspectos elencados por Goodstadt et al. (8) acerca dos critérios estabelecidos para os programas, pode-se afirmar que os mesmos apresentaram elementos que vão ao encontro da promoção da saúde no envelhecimento, apesar de não terem sido avaliados na li- teratura estudada com comprovação da eficácia de suas ações.

Destaca-se que os programas analisados de promoção da saúde de idosos tiveram como alicerce o empoderamento, por tornar o idoso protagonista e atuante nas escolhas que determinam seu estado de saúde e inserção na sua teia social. Tal empoderamento foi concretizado pela criação de um ambiente de suporte para a prevenção de agravos, aumento da capacidade funcional e melhoria da qualidade de vida, traduzido pelo que podemos cha- mar de conceito ampliado de saúde. Finalmente, deve-se ressaltar que a produção de evidências da promoção da saúde deve ser pauta das agendas tanto dos idealizadores como dos implementadores de políticas, programas e projetos relacionados ao processo de envelhecimento.

Agradecimentos. $\mathrm{O}$ presente trabalho recebeu apoio da Coordenação de Aperfeiçoamento de Pessoal de Nível Superior (CAPES) (processo n ${ }^{\circ} 23038.009788 / 2010-$ 78, AUX-PE-Pró-Ensino Saúde 2034/2010).

\section{REFERÊNCIAS}

1. Veras R. Envelhecimento populacional contemporâneo: demandas, desafios e inovações. Rev Saude Publica. 2009;43(3):548-44.

2. Veras R. A novidade da agenda social contemporânea: a inclusão do cidadão de mais idade. A Terceira idade. 2003;14(28):6-29.

3. Sousa VC, Saldanha AA, Araújo LF. Viver com AIDS na terceira idade. Em: Congresso Virtual HIV/AIDS. 2006;7. Disponível em: http://aidscongress.net/Modules/WebC AidsCongress/CommunicationHTML.aspx? $\mathrm{Mid}=36 \&$ CommID=324. Acessado em $22 \mathrm{de}$ abril de 2010.

4. Pereira RJ, Cotta RM, Franceschini SC, Ribeiro RC, Sampaio RF, Priore SE, et al. Influência de fatores sociossanitários na qualidade de vida dos idosos de um município do Sudeste do Brasil. Cienc Saude Coletiva. Disponível em: cienciaesaudecoletiva.com.br/artigos/artigo int.php?id_artigo=3374. Acesso em 24 de Junho de 2010.

5. Pereira RJ, Cotta RM, Franceschini SC, Ribeiro RC, Sampaio RF, Priore SE, et al. Contribuição dos domínios físico, social, psicológico e ambiental para a qualidade de vida global de idosos. Rev Psiquiatr Rio Gd Sul. 2006;28(1): 27-38.

6. Brasil, Ministério da Saúde. Portaria 2 528/ 2006. Disponível em: http://www.brasilsus. com.br/legislacoes/gm/1363-2528.html?q=. Acessado em 5 de junho de 2011.

7. Assis M, Hartz ZM, Valla VV. Programas de promoção da saúde do idoso: uma revisão da literatura científica no período de 1990 a 2002. Cienc Saude Coletiva. 2004;9(3):557-81.

8. Goodstadt MS, Hyndman B, McQueen DV, Potvin L, Rootman I, Springett J. Evaluation in health promotion: synthesis and recommendations. WHO Reg Publ Eur Ser. 2001; (92):517-33.

9. Teixeira MB. Empoderamento de idosos em grupo voltado à promoção da saúde [disser- tação]. Rio de Janeiro: Escola Nacional de Saúde Pública; 2002.

10. Cabral BE, Nóbrega CV, Silva KQ, Calado LR, Cartaxo RM. Programa Interdisciplinar de Apoio à Terceira Idade. Anais do $2^{\circ}$ Congresso Brasileiro de Extensão Universitária. Belo Horizonte, 12 a 15 de setembro de 2004. Disponível em: http://ufcg.edu.br/ piat/ download/Anais_2Congresso. Acessado em maio de 2011.

11. Cabral BE, org. Programa interdisciplinar de apoio à terceira idade - PIATI. Anais do II Encontro de Extensão da UFCG. Campina Grande, 30 novembro de 2004. Disponível em: http://ufcg.edu.br/ piat/download/II ExtensaoUFCG. Acessado em 5 de junho de 2011.

12. Diogo MJ, Ceolim MF, Cintra FA. Implantação do grupo de atenção à Saúde do idoso (GRASI), no Hospital de Clínicas na Universidade Estadual de Campinas (SP): relato de experiência. Rev Latinoam Enferm. 2000;8(5):85-90.

13. Veras R, Caldas CP. UNATI-UERJ - 10 anos, um modelo de cuidado integral para a população que envelhece. Rio de Janeiro: Universidade Federal do Rio de Janeiro; 2004.

14. Fletcher A, Breeze E, Walters R. Health promotion for the older people: what are the opportunities? Promot Educ. 1999;6(4):4-7.

15. Assis M, Hartz ZM, Pacheco LC, Valla VV. Avaliação do projeto de promoção da saúde do núcleo de atenção ao idoso: um estudo exploratório. Interface - Comunic, Saude, Educ. 2009;13(29):367-82.

16. Barreto KML. Universidade Aberta à Terceira Idade (UnATI/UFPE): um perfil sócioepidemiológico dos participantes [dissertação]. Recife: Fundação Oswaldo Cruz, Instituto Aggeu Magalhães, Departamento de Saúde Coletiva - NESC; 1999.

17. Organização Pan-Americana da Saúde. Carta de Ottawa. Primeira Conferência Internacio- nal sobre Promoção da Saúde. Disponível em: http://www.opas.org.br/promocao/upload Arq/Ottawa.pdf. Acessado em 6 de junho de 2011.

18. Martins PC, Cotta RM, Siqueira-Batista R, Mendes FF, Franceschini SC, Priore SE, et al. Democracia e empoderamento no contexto da promoção da saúde: possibilidades e desafios apresentados ao Programa de Saúde da Família. Physis (Rio J). 2009;19(3):679-94.

19. Sen A. ¿Por qué la equidade en salud? Pan American J Public Health. 2002;11(5-6):302-9.

20. Freire P. Pedagogia do oprimido. São Paulo: Paz e Terra; 2004.

21. Unión Internacional de Promoción y Educación para la Salud [UIPES]. La evidencia de la eficacia de la promoción de la salud. Configurando la salud pública en una nueva Europa. Informe de la UIPES para la Comisión Europea. Madri: Evidence Book; 1999.

22. Campos GW, Barros RB, Castro AM. Avaliação de política nacional de promoção da saúde. Cienc Saude Coletiva. 2004;9(3):745-9.

23. Bosi ML, Uchimura KY. Avaliação da qualidade ou avaliação qualitativa do cuidado em saúde. Rev Saude Publica. 2007;41(1):150-3.

24. Demo P. Educação e qualidade. Campinas: Papirus; 2000.

25. Marinho A, Façanha LO. Programas sociais: efetividade, eficiência e eficácia como dimensões operacionais da avaliação. Rio de Janeiro: IPEA, 2001. (Texto para discussão, 787).

26. Araujo MA. Responsabilização pelo controle de resultados no Sistema Único de Saúde no Brasil. Rev Panam Salud Publica. 2010;27(3): 230-6.

Manuscrito recebido em 27 de julho de 2010. Aceito em versão revisada em 26 de fevereiro de 2011. 
ABSTRACT Objective. To search the literature for evidence of the contribution of elderly health programs to the process of healthy aging in Brazil.

Evidence of the contribution of elderly support programs to healthy aging in Brazil
Methods. The databases Science Direct, SciELO, LILACS, IBECS, the Cochrane Library, and MEDLINE were searched to find articles focusing on initiatives developed for the elderly population and to promote healthy aging, providing a multidimensional evaluation of the quality of life of the elderly, and covering aspects relating to specific health promotion practices. The initiatives were considered effective if the following criteria were met: having goals that included the improvement of health and well-being, having a focus on holistic health, using empowerment as a central mechanism to improve health, and meeting the prerequisites for health and developing actions in the priority areas described in the Ottawa Charter.

Results. Ten articles referring to five elderly health promotion programs in Brazil were identified. All the programs met the efficacy criteria, and included elements that promoted healthy aging through characteristics that fostered the insertion of the elderly in society through the creation of a support environment for the prevention of injury, increase in functional capacity, and improvement of quality of life.

Conclusions. Studies are required to produce scientific evidence on the efficacy, efficiency, and effectiveness of elderly health programs to determine the value of these actions to promote healthy aging.

Key words Evidence-based practice; health promotion; aging; Brazil. 